\title{
Research Article \\ Switched Quantization Level Control of Networked Control Systems with Packet Dropouts
}

\author{
Shuo Wang, Mei Yu, Hangfei Wang, and Wen Tan \\ School of Control and Computer Engineering, North China Electric Power University, Beijing 102206, China \\ Correspondence should be addressed to Mei Yu; meiyu@ncepu.edu.cn
}

Received 2 December 2013; Revised 28 January 2014; Accepted 30 January 2014; Published 12 March 2014

Academic Editor: Yang Shi

Copyright (C) 2014 Shuo Wang et al. This is an open access article distributed under the Creative Commons Attribution License, which permits unrestricted use, distribution, and reproduction in any medium, provided the original work is properly cited.

\begin{abstract}
This paper investigates the relationship between the maximum allowable dropout bound and the quantization density. Networked Control System (NCS) is described as a time-delay switched system with constrained switching signals. A switched dynamic output feedback controller with prescribed disturbance attenuation level is designed via a cone complement linearization approach. A novel stability criterion is obtained by switched system theory. Furthermore, finding an appropriate quantization density used when packet dropout occurs is converted to an optimization problem.
\end{abstract}

\section{Introduction}

Networked Control System (NCS) has been widely used in industrial production such as aerospace engineering, robotic engineering, and power system. The control information is exchanged via communication networks in NCS. It has many attractive advantages, such as less wiring, low costs, ease of system diagnosis and maintenance, and increased flexibility. On the contrary, it may induce some problems such as quantization error and packet dropouts which deteriorate the performance of system or even cause instability [1].

Quantization effect cannot be avoided in the digital control process, and the stabilization problem by quantized feedback has been extensively studied in the last few years [2-5]. In [6], a sufficient condition is presented for filtering error system to be mean square exponentially stable with prescribed $H_{\infty}$ performance by employing the multiple Lyapunov function method. The obtained condition depends on packet dropout rate of NCS. In [7], coarsest memoryless quantizer which can stabilize a single-input system with packet loss is proposed in the sense of stochastic quadratic stability. All of the aforementioned works use time-invariant signal quantization strategy and ignore network load condition. Actually, it is less conservative to switch quantization level according to network load condition. To achieve this, [8] proposes an adaptive quantization density method to reduce network congestion. The quantization density is designed to be a function of the networked load modeled by a Markov process. However, no specific parameters reflecting network load has been introduced. The Markov chain may not be obtained easily and might vary under some unknown disturbance. Packet loss is an important indicator for load condition and it can be measured easily by adding a counter at controller node. Therefore, packet loss number is treated as switching signal of load condition in our paper. A large packet loss number means that the network load condition is worse. A coarser quantization density which implies transmitting less information can decrease the incidence rates of packet drop. So the quantization density should be increased as much as possible in order to exchange for better network performance when successive packet dropouts occur. Nevertheless, the quantization density cannot be increased indefinitely for a specified number of packet loss because this may cause oscillation in system [9-12]. Hence, it is essential to investigate the relationship between packet loss number and quantization level.

Many researchers have studied packet loss in NCS and lots of stability analysis results have been obtained [13-16]. For most of the aforementioned papers about packet loss problem, stochastic system approach is usually adopted to investigate the stability criterion of NCS. Generally, packet dropout process is modeled as an independent and identical 


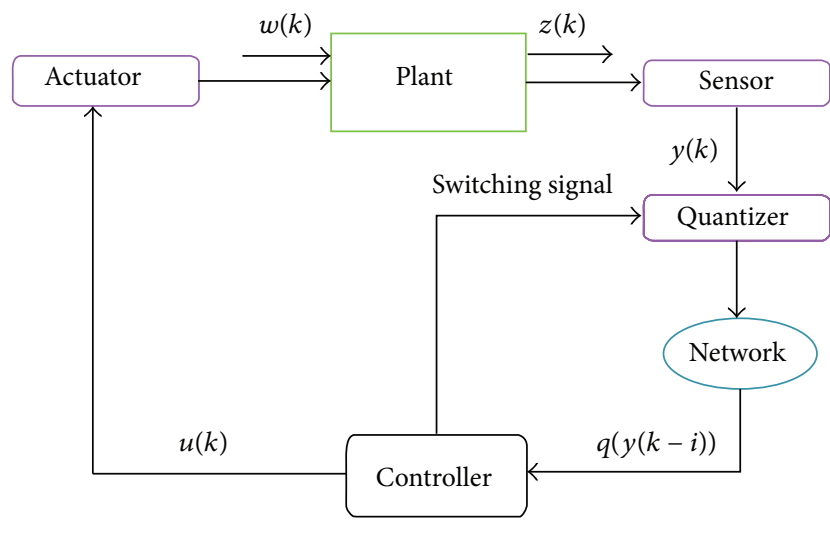

Figure 1: Structure of NCS.

distributed Bernoulli process [17-20]. It is easy to study the relationship between packet loss rate and quantization density by this method. However, this makes it difficult to add successive packet dropout number to the NCS model. The transition probability matrix or packet loss probability must be known beforehand by experimentation. The controller designed via stochastic system approach is constrained by packet loss probability. Once the probability changes, the controller should be redesigned, which reduces the flexibility. The quantitative relationship between packet dropouts number and quantization density has not been studied by switched system approach yet. Switched system approach is more flexible and independent of packet loss probability. This inspires us to try modeling the NCS as a switched linear discrete-time system.

In this paper, the switched quantization strategy is set as follows: if the successive packet drop number increases, coarser quantization densities will be used at the next sampling time to relieve network congestion; a fine quantization density is selected when the data is transmitted successfully. An algorithm of a dynamic output feedback controller synthesis is proposed. A stability criterion dependent on quantization density and successive packet dropout number is given. The relationship between number of packet dropouts and quantization density is established through switched system theory. The structure of NCS proposed in this paper is depicted in Figure 1.

The advantage of the method presented in this paper is that the probability of packet loss need not be prior informed. Less experiments about network load mean that the costs of test and lots of time can be saved. The load condition of network can be dynamically optimized by transmitting less information when packet dropout happens. Fewer inequalities need to be solved since the constrained switching signal approach [21] is used to model the packet dropout. This greatly reduces the computation.

Two assumptions are needed for designing the controller and quantization density as follows: (1) the network locates between the sensor and controller node; (2) if packet loss occurs, the actuator will use the last successful transmitted data.
This paper is organized as follows. Section 2 gives the system description, the form of dynamic output feedback controller, and quantization error model. Main results for controller synthesis and stability analysis are presented in Section 3. The validity of the approach is illustrated by a simulation example in Section 4. In Section 5, a summary for this paper is proposed.

\section{System Modeling and Problem Formulation}

Consider a discrete-time linear system model

$$
\begin{gathered}
x(k+1)=A x(k)+B_{1} w(k)+B_{2} u(k), \\
z(k)=C_{1} x(k)+D_{1} w(k), \\
y(k)=C_{2} x(k),
\end{gathered}
$$

where $x(k) \in \mathfrak{R}^{n}, u(k) \in \mathfrak{R}^{m}, z(k) \in \mathfrak{R}^{m_{1}}, y(k) \in \mathfrak{R}^{m_{2}}$ are the state vector, input, controlled output, and measured output. $w(k)$ is the disturbance which belongs to $l_{2}[0, \infty)$. $A, B_{1}, B_{2}, C_{1}, C_{2}, D_{1}$ are known matrices with appropriate dimensions.

When the number of packet dropouts increases, the quantization density should be switched to reduce the network congestion. The logarithmic quantizer is proposed as follows:

$$
\begin{aligned}
& q(\nu, \sigma) \\
& = \begin{cases}\rho^{h}(\sigma) & \text { if } \frac{1}{1+\delta(\sigma)} \rho^{h}(\sigma)<\nu \\
& \quad \leq>0, h=0, \pm 1, \pm 2, \ldots \\
0 & \text { if } \nu=0, \\
-q(-\nu, \sigma) & \text { if } \nu<0,\end{cases}
\end{aligned}
$$

where $v$ is the signal to be quantized and $q(\nu, \sigma)$ is the quantized signal. The introduction of variable $\sigma$ aims to represent the switching signal of quantization density. $0<$ $\rho(\sigma)<1$ is related to $\delta(\sigma)$ by

$$
\delta(\sigma)=\frac{1-\rho(\sigma)}{1+\rho(\sigma)} .
$$

The associated quantized set $\mathscr{U}$ is given as

$$
\mathcal{U}=\left\{ \pm \rho^{h}(\sigma), h=0, \pm 1, \pm 2, \ldots\right\} \cup\{0\} .
$$

The quantization error $e(k, \sigma)$ is proposed as follows:

$$
\begin{gathered}
e(k, \sigma)=q(\nu(k), \sigma)-\nu(k)=\Delta_{q}(\sigma) \nu(k), \\
\Delta_{q}(\sigma) \in[-\delta(\sigma), \delta(\sigma)], \\
\sigma \in\{0,1, \ldots, d\},
\end{gathered}
$$


where $d$ is the maximum number of packet loss. According to the definition of $\Delta_{q}(\sigma)$, it can be treated as norm-bounded time-varying uncertain parameter in NCS [22]. This means that

$$
\Delta_{q}(\sigma)=H F E
$$

where

$$
\begin{gathered}
H=1, \quad E=\delta(\sigma), \\
F^{T} F \leq I .
\end{gathered}
$$

A switched dynamic output feedback (DOF) controller is used to compensate the packet dropouts as follows:

$$
\begin{gathered}
\widehat{x}(k+1)=A_{c}(i) \hat{x}(k)+B_{c}(i) q(y(k-i)), \\
u(k)=C_{c}(i) \hat{x}(k) .
\end{gathered}
$$

Remark 1. The variable $i$ has two different meanings in the system. It stands for: (a) the switching signal of controller; (b) the successive dropout number of networked control system at instant $(k+1) T$. The switching signal of quantizer is a map, $\sigma: \mathbb{Z}^{+} \mapsto\{0,1, \ldots, d\}$.

Remark 2. In this paper, the switching signal $i$ is not arbitrary, but satisfies the following constraints. If the successive packet dropout number is $i-1(i \in\{1,2, \ldots, d\})$ at the present time, the number of consecutive packet loss is equal to $i$ or 0 at the next instant; if the packet drop number is $d$ at this instant, the data must be transmitted successfully at next sampling time.

Now define a new state vector $\xi(k)=\left[x^{T}(k), \widehat{x}^{T}(k)\right]^{T}$. From (1), (5), and (8), the closed-loop system is given as follows:

$$
\begin{gathered}
\xi(k+1)=A_{\mathrm{cl}}(i) \xi(k)+B_{\mathrm{cl}}(i)\left(1+\Delta_{q}(\sigma)\right) \bar{C}_{2} \xi(k-i) \\
+\bar{B}_{1} w(k), \\
z(k)=\bar{C}_{1} \xi(k)+D_{1} w(k),
\end{gathered}
$$

where $A_{\mathrm{cl}}(i)=\left[\begin{array}{cc}A & B_{2} C_{c}(i) \\ 0 & A_{c}(i)\end{array}\right], B_{\mathrm{cl}}(i)=\left[\begin{array}{c}0 \\ B_{c}(i)\end{array}\right], \bar{B}_{1}=\left[\begin{array}{c}B_{1} \\ 0\end{array}\right], \bar{C}_{2}=$ $\left[\begin{array}{ll}C_{2} & 0\end{array}\right], \bar{C}_{1}=\left[\begin{array}{ll}C_{1} & 0\end{array}\right]$.

The problem under investigation is formulated as follows.

(i) For a prescribed $\gamma>0$ and quantization densities $\delta(\sigma)$, design a switched DOF controller of the form (8) such that:

(1) the system (9) is asymptotically stable with $w(k)=0$

(2) under the zero-initial condition, the controlled output $z(k)$ satisfies

$$
\sum_{k=0}^{\infty} z^{T}(k) z(k) \leq \gamma^{2} \sum_{k=0}^{\infty} w^{T}(k) w(k)
$$

for all nonzero $w(k)$. (ii) For an $H_{\infty}$ controller obtained by the method above, select a maximum $\delta(\sigma)$ in order to ease network congestion.

The following lemmas will play a key role in designing the DOF controller for NCS.

Lemma 3 (see [23]). Define two new vectors: $\tilde{\xi}(k)=\xi(k+1)-$ $\xi(k), \eta(k)=\left[\xi^{T}(k), \xi^{T}(k-i), w^{T}(k)\right]^{T} \in \mathfrak{R}^{4 n+1} ;$ then for any matrices $R \in \mathfrak{R}^{2 n \times 2 n}, M \in \mathfrak{R}^{2 n \times(4 n+1)}$, and $Z \in \mathfrak{R}^{(4 n+1) \times(4 n+1)}$ satisfying

$$
\left[\begin{array}{cc}
R & M \\
M^{T} & Z
\end{array}\right] \geq 0
$$

the following inequality holds

$$
-\sum_{j=k-i}^{k-1} \widetilde{\xi}^{T}(j) R \widetilde{\xi}(j) \leq \eta^{T}(k)\left\{\Upsilon_{1}+\Upsilon_{1}^{T}+i Z\right\} \eta(k),
$$

where $\Upsilon_{1}=M^{T}[I,-I, 0]$.

Lemma 4 (see [24]). Assume that $H, F, E$ are real matrices with appropriate dimensions and $F^{T} F \leq I$. The inequality $Y+$ $H F E+E^{T} F^{T} H^{T}<0$ holds if there exists a scalare $>0$ satisfying

$$
Y+\varepsilon^{-1} E^{T} E+\varepsilon H H^{T}<0
$$

\section{Controller Synthesis and Stability Analysis}

The following theorem provides a methodology of designing a switched output feedback controller.

Theorem 5. For a given scalar $\gamma>0$ and quantization densities $\delta(\sigma)$, if there exist positive symmetric matrices $P(i)>$ $0, \mathscr{P}(i)>0, P(0)>0, \mathscr{P}(0)>0$, matrices $R, R_{1} \in \mathfrak{R}^{2 n \times 2 n}$, $M_{1} \in \mathfrak{R}^{2 n \times 2 n}, M_{2} \in \mathfrak{R}^{2 n \times 2 n}, M_{3} \in \mathfrak{R}^{2 n \times 1}, Z_{11} \in \mathfrak{R}^{2 n \times 2 n}$, $Z_{12} \in \Re^{2 n \times 2 n}, Z_{13} \in \mathfrak{R}^{2 n \times 1}, Z_{22} \in \mathfrak{R}^{2 n \times 2 n}, Z_{23} \in \mathfrak{R}^{2 n \times 1}$, $Z_{33} \in \Re^{1 \times 1}, A_{c}(0), B_{c}(0), C_{c}(0), A_{c}(i), B_{c}(i), C_{c}(i)$, scalar $\varepsilon>0$ satisfying the following inequalities for all $i \in\{1, \ldots, d\}$ :

$$
\left[\begin{array}{cccc}
R_{1} & M_{1} & M_{2} & M_{3} \\
* & Z_{11} & Z_{12} & Z_{13} \\
* & * & Z_{22} & Z_{23} \\
* & * & * & Z_{33}
\end{array}\right] \geq 0
$$

$$
\left[\begin{array}{cccc}
\Phi(i-1) & * & * & * \\
\Gamma_{1}(i) & -\mathscr{P}(i) & * & * \\
i \Gamma_{2}(i) & 0 & -i R & * \\
0 & \bar{C}_{2}^{T} B_{\mathrm{cl}}^{T}(i) & i \bar{C}_{2}^{T} B_{\mathrm{cl}}^{T}(i) & -\varepsilon^{-1} I
\end{array}\right]<0
$$

$$
\begin{aligned}
& {\left[\begin{array}{ccc}
\Lambda(i) & * & * \\
\Pi_{1}(0) & -\mathscr{P}(0) & * \\
0 & \bar{C}_{2}^{T} B_{\mathrm{cl}}^{T}(0) & -\varepsilon^{-1} I
\end{array}\right]<0,} \\
& {\left[\begin{array}{ccc}
\Lambda(0) & * & * \\
\Pi_{1}(0) & -\mathscr{P}(0) & * \\
0 & \bar{C}_{2}^{T} B_{\mathrm{cl}}^{T}(0) & -\varepsilon^{-1} I
\end{array}\right]<0,}
\end{aligned}
$$


where

$$
\begin{aligned}
& \Phi(i-1)=\left[\begin{array}{ccc}
-P(i-1)+\bar{C}_{1}^{T} \bar{C}_{1}+M_{1}+M_{1}^{T}+i Z_{11} & M_{2}-M_{1}^{T}+i Z_{12} & \bar{C}_{1}^{T} D_{1}+M_{3}+i Z_{13} \\
* & \varepsilon^{-1} \delta^{2}(d) I-M_{2}-M_{2}^{T}+i Z_{22} & i Z_{23}-M_{3} \\
* & * & D_{1}^{T} D_{1}-\gamma^{2}+i Z_{33}
\end{array}\right], \\
& \Gamma_{1}(i)=\left[\begin{array}{lll}
A_{\mathrm{cl}}(i) & B_{\mathrm{cl}}(i) \bar{C}_{2} & \bar{B}_{1}
\end{array}\right] \\
& \Gamma_{2}(i)=\left[\begin{array}{lll}
A_{\mathrm{cl}}(i)-I & B_{\mathrm{cl}}(i) \bar{C}_{2} & \bar{B}_{1}
\end{array}\right] \\
& \Lambda(i)=\left[\begin{array}{cc}
-P(i)+\bar{C}_{1}^{T} \bar{C}_{1}+\varepsilon^{-1} \delta^{2}(i) I & \bar{C}_{1}^{T} D_{1} \\
* & D_{1}^{T} D_{1}-\gamma^{2}
\end{array}\right], \\
& \Pi_{1}(0)=\left[\begin{array}{ll}
A_{\mathrm{cl}}(0)+B_{\mathrm{cl}}(0) \bar{C}_{2} & \bar{B}_{1}
\end{array}\right], \\
& P(i) \mathscr{P}(i)=I, \quad P(0) \mathscr{P}(0)=I, \quad R_{1} R=I,
\end{aligned}
$$

then the closed-loop system is stable with the prescribed $H_{\infty}$ performance.

Proof. Construct a switched Lyapunov function $V(k)$ for the closed-loop system as

$$
V(k)=\xi^{T}(k) P(i-1) \xi(k)+\sum_{l=-i}^{-1} \sum_{j=k+l}^{k-1} \widetilde{\xi}^{T}(j) R_{1} \widetilde{\xi}(j)
$$

Case 1. Assume that at times $k T$ and $(k+1) T$, the numbers of packet loss are $i-1$ and $i(1 \leq i \leq d)$; then the forward difference of $V(k)$ is given as follows:

$$
\begin{aligned}
\Delta V(k)= & \xi^{T}(k+1) P(i) \xi(k+1)-\xi^{T}(k) P(i-1) \xi(k) \\
& +i \widetilde{\xi}^{T}(k) R_{1} \widetilde{\xi}(k)-\sum_{j=k-i}^{k-1} \widetilde{\xi}^{T}(j) R_{1} \tilde{\xi}(j) .
\end{aligned}
$$

Using Lemma 3, the following inequality can be obtained:

$$
\begin{aligned}
\Delta V(k)= & \eta^{T}(k) \widetilde{\Gamma}_{1}^{T}(i) P(i) \widetilde{\Gamma}_{1}(i) \eta(k)-\xi^{T}(k) P(i-1) \xi(k) \\
& +i \eta^{T}(k) \widetilde{\Gamma}_{2}^{T}(i) R_{1} \widetilde{\Gamma}_{2}(i) \eta(k)-\sum_{j=k-i}^{k-1} \widetilde{\xi}^{T}(j) R_{1} \widetilde{\xi}(j) \\
\leq & \eta^{T}(k) \widetilde{\Gamma}_{1}^{T}(i) P(i) \widetilde{\Gamma}_{1}(i) \eta(k)-\xi^{T}(k) P(i-1) \xi(k) \\
& +i \eta^{T}(k) \widetilde{\Gamma}_{2}^{T}(i) R_{1} \widetilde{\Gamma}_{2}(i) \eta(k) \\
& +\eta^{T}(k)\left(\Upsilon_{1}+\Upsilon_{1}^{T}+i Z\right) \eta(k)
\end{aligned}
$$

$$
\begin{aligned}
& +\eta^{T}(k) \Psi^{T} \Psi \eta(k)-\gamma^{2} w^{T}(k) w(k) \\
& -z^{T}(k) z(k)+\gamma^{2} w^{T}(k) w(k)
\end{aligned}
$$

where

$$
\begin{gathered}
\Upsilon_{1}=M^{T}[I,-I, 0], \\
\widetilde{\Gamma}_{1}(i)=\left[A_{\mathrm{cl}}(i), B_{\mathrm{cl}}(i)\left(1+\Delta_{q}(\sigma)\right) \bar{C}_{2}, \bar{B}_{1}\right], \\
\widetilde{\Gamma}_{2}(i)=\left[A_{\mathrm{cl}}(i)-I, B_{\mathrm{cl}}(i)\left(1+\Delta_{q}(\sigma)\right) \bar{C}_{2}, \bar{B}_{1}\right], \\
\Psi=\left[\bar{C}_{1}, 0, D_{1}\right] .
\end{gathered}
$$

Partition matrices $M$ and $Z$ as $M=\left[M_{1}, M_{2}, M_{3}\right], Z=$ $\left[\begin{array}{ccc}Z_{11} & Z_{12} & Z_{13} \\ * & Z_{22} & Z_{23} \\ * & * & Z_{33}\end{array}\right]$

Applying Schur complement (see [25]) on (21), $\Delta V(k) \leq$ $-z^{T}(k) z(k)+\gamma^{2} w^{T}(k) w(k)$ is equivalent to the following matrix inequality:

$$
\left[\begin{array}{ccc}
\widetilde{\Phi}(i-1) & * & * \\
\widetilde{\Gamma}_{1}(i) & -P^{-1}(i) & * \\
i \widetilde{\Gamma}_{2}(i) & 0 & -i R_{1}^{-1}
\end{array}\right]<0,
$$


where

$$
\widetilde{\Phi}(i-1)=\left[\begin{array}{ccc}
-P(i-1)+\bar{C}_{1}^{T} \bar{C}_{1}+M_{1}+M_{1}^{T}+i Z_{11} & * & * \\
-M_{1}+M_{2}^{T}+i Z_{12}^{T} & -M_{2}-M_{2}^{T}+i Z_{22} & * \\
D_{1}^{T} \bar{C}_{1}+M_{3}^{T}+i Z_{13}^{T} & -M_{3}^{T}+i Z_{23}^{T} & D_{1}^{T} D_{1}-\gamma^{2}+i Z_{33}
\end{array}\right]
$$

From (6), we know that

$$
B_{\mathrm{cl}}(i) \Delta_{q}(\sigma) \bar{C}_{2}=B_{\mathrm{cl}}(i) F \delta(\sigma) \bar{C}_{2}
$$

Rewriting inequality (23), we can note that

$$
\begin{aligned}
& {\left[\begin{array}{ccc}
\widetilde{\Phi}(i-1) & * & * \\
\Gamma_{1}(i) & -P^{-1}(i) & * \\
i \Gamma_{2}(i) & 0 & -i R^{-1}
\end{array}\right]+\left[\begin{array}{c}
0 \\
\widetilde{B}_{\mathrm{cl}}(i) \\
i \widetilde{B}_{\mathrm{cl}}(i)
\end{array}\right] F[\delta(\sigma) \widetilde{I} \quad 0 \quad 0]} \\
& +\left[\begin{array}{c}
\delta(\sigma) \widetilde{I}^{T} \\
0 \\
0
\end{array}\right] F^{T}\left[\begin{array}{lll}
0 & \widetilde{B}_{\mathrm{cl}}^{T}(i) & \left.i \widetilde{B}_{\mathrm{cl}}^{T}(i)\right]<0,
\end{array}\right.
\end{aligned}
$$

where

$$
\widetilde{I}=\left[\begin{array}{lll}
0 & I & 0
\end{array}\right], \quad \widetilde{B}_{\mathrm{cl}}(i)=B_{\mathrm{cl}}(i) \bar{C}_{2} .
$$

Applying Lemma 4 and Schur complement on the inequality above, (15) is easy to be obtained according to $\Delta_{q}(\sigma) \leq \delta(\sigma) \leq$ $\delta(d)$. This guarantees inequality (23). Adding $\Delta V(k)$ from 0 to $\infty$, the following result can be derived owing to the zeroinitial condition $(V(0)=0)$ :

$$
V(\infty)-V(0) \leq \sum_{k=0}^{\infty}-z^{T}(k) z(k)+\gamma^{2} w^{T}(k) w(k)
$$

This yields

$$
\sum_{k=0}^{\infty} z^{T}(k) z(k) \leq \gamma^{2} \sum_{k=0}^{\infty} w^{T}(k) w(k)
$$

When $w(k)=0$, it is obvious that $\Delta V(k) \leq 0$.

Case 2. At time $k T$, the packet dropout number is $i$ or 0 . At time $(k+1) T$, the packet is transmitted successfully. In this case, the second term of the Lyapunov functional becomes zero. Eliminate $\xi(k-i)$ in $\eta(k)$. Replacing $P(i)$ in $(21)$ with $P(0)$ and $P(i-1)$, the proof is almost the same with that of Case 1 . We can see that inequalities (16) and (17) guarantee the stabilization of NCS with prescribed $H_{\infty}$ performance.
A switched controller can be obtained by solving the Bilinear Matrix Inequalities in Theorem 5. A cone complement approach shown as follows can be used to solve the problem [26]:

$$
\begin{array}{ll}
\min & \operatorname{Tr}\left(R_{1} R+\sum_{i=0}^{d} P(i) \mathscr{P}(i)\right) \\
\text { subject to } & (14),(15),(16),(17) \text { and } \\
& {\left[\begin{array}{cc}
P(i) & I \\
I & \mathscr{P}(i)
\end{array}\right] \geq 0 \quad(i \in\{0,1, \ldots, d\}),} \\
& {\left[\begin{array}{cc}
R_{1} & I \\
I & R
\end{array}\right] \geq 0 .}
\end{array}
$$

For given controller matrices of system (9), a method of stability analysis is also needed in the sense of time-variant quantization density. Therefore, a novel stability criterion for system (9) with switched quantization level is proposed as follows. This method can also be used in choosing a suitable quantization density.

Theorem 6. For a prescribed scalar $i(i=1, \ldots, d), \gamma>0$, controller matrices $A_{c}(0), B_{c}(0), C_{c}(0), A_{c}(i), B_{c}(i), C_{c}(i)$, and quantization densities $\delta(\sigma)$, if there exist sets of symmetric positive-definite matrices $P(i)>0, P(0)>0$, matrices $R_{1}, Z_{11}$, $Z_{12}, Z_{13}, Z_{22}, Z_{23}, Z_{33}, M_{1}, M_{2}, M_{3}$, scalar $\varepsilon>0$ satisfy the following inequalities:

$$
\begin{gathered}
{\left[\begin{array}{cccc}
R_{1} & M_{1} & M_{2} & M_{3} \\
* & Z_{11} & Z_{12} & Z_{13} \\
* & * & Z_{22} & Z_{23} \\
* & * & * & Z_{33}
\end{array}\right] \geq 0,} \\
{\left[\begin{array}{ccc}
\Theta_{11} & \Theta_{12} & \Theta_{13} \\
* & \Theta_{22} & \Theta_{23} \\
* & * & \Theta_{33}
\end{array}\right]<0,} \\
{\left[\begin{array}{cc}
\Xi_{11} & \Xi_{12} \\
* & \Xi_{22}
\end{array}\right]<0,} \\
{\left[\begin{array}{cc}
\widetilde{\Xi}_{11} & \Xi_{12} \\
* & \Xi_{22}
\end{array}\right]<0,}
\end{gathered}
$$


where

$$
\begin{gathered}
\Theta_{11}=\Phi(i-1), \\
\Theta_{12}=\Gamma_{1}^{T}(i) P(i), \\
\Theta_{13}=\left[\begin{array}{ll}
i \Gamma_{2}^{T}(i) R_{1} & 0
\end{array}\right], \\
\Theta_{22}=-P(i), \\
\Theta_{23}=\left[\begin{array}{lll}
0 & P(i) B_{\mathrm{cl}}(i) \bar{C}_{2}
\end{array}\right], \\
\Theta_{33}=\left[\begin{array}{cc}
-i R_{1} & i R_{1} B_{\mathrm{cl}}(i) \bar{C}_{2} \\
* & -\mathcal{\varepsilon}^{-1} I
\end{array}\right], \\
\Xi_{11}=\Lambda(i), \\
\Xi_{12}=\left[\begin{array}{ccc}
\Pi_{1}^{T} & (0) P(0) & 0
\end{array}\right], \\
\Xi_{22}=\left[\begin{array}{cc}
-P(0) & P(0) B_{\mathrm{cl}}(i) \bar{C}_{2} \\
* & -\varepsilon^{-1} I
\end{array}\right], \\
\widetilde{\Xi}_{11}=\Lambda(0),
\end{gathered}
$$

the closed-loop system is asymptotically stable with the disturbance attenuation level $\gamma$.

Proof. Consider Case 1 in the proof of Theorem 5. Multiply (15) to the right by the matrix $\operatorname{diag}\left\{I, P(i), R_{1}, I\right\}$ and the left by its transpose. By Schur complement, if the inequalities (31) and (32) hold, then the inequality (23) holds [27]. Thus the closed-loop system is asymptotically stable with the disturbance attenuation level $\gamma$.

Case 2: Pre- and postmultiply (16) and (17) by $\operatorname{diag}\{I, P(0), I\}$; then if (33) and (34) hold, the closedloop system is asymptotically stable with the disturbance attenuation level $\gamma$. The proof is similar to that of Case 1 .

After designing a switched $H_{\infty}$ controller, a $\delta(d)$ that is large enough should be selected to reduce the packet dropouts. This can be converted to a convex optimization problem as follows:

$\max \quad \delta(d)$

subject to inequalities (31), (32), (33), (34) in Theorem 6.

Remark 7. The quantization density for the worst packet loss case $\delta(d)$ can be increased until the LMIs are infeasible. The maximum $\delta(d)$ can be used to quantize the data transmitted to the controller when the load condition takes a turn for the worse. By the approach proposed in this paper, we not only design a less conservative controller by changing quantization level when network load is heavy but also search for an optimized quantization density.

\section{Numerical Examples}

In this section, the numerical simulation is given to illustrate the main results in this paper.

Consider the following example of a discrete-time LTI system (1), where

$$
\begin{gathered}
A=\left[\begin{array}{cccc}
0.08 & -0.025 & 0 & 1 \\
1 & 0 & 0 & 0 \\
-0.8 & 0.5 & 0.002 & -0.03 \\
0 & 0 & 0.0792 & 0
\end{array}\right], \quad B_{1}=\left[\begin{array}{c}
0 \\
0.1 \\
0 \\
0.05
\end{array}\right], \\
B_{2}=\left[\begin{array}{l}
1 \\
0 \\
0 \\
0
\end{array}\right], \quad C_{1}=\left[\begin{array}{llll}
0 & 0.1 & 0 & 0
\end{array}\right], \\
C_{2}=\left[\begin{array}{llll}
4.5 & -4.5 & 0 & 2
\end{array}\right], \quad D_{1}=0.03 .
\end{gathered}
$$

The maximum number of packet loss is assumed to be 3. For the simplicity of presentation, the switched output feedback controller is designed to have two modes $\{0,1\}$. 0 represents that there is no packet dropout at this time, whereas 1 means that packet loss happens. Set the initial value of quantization density as $\delta(0)=0.02, \delta(1)=0.04 . \gamma=0.95$ is chosen for the design of controller. Using the approach given in Theorem 5, the controller matrices are obtained as follows:

$$
\begin{aligned}
& A_{c}(0)=\left[\begin{array}{cccc}
-0.0304 & -0.1710 & -0.1544 & 0.0041 \\
0.1589 & 0.1676 & 0.0222 & -0.1436 \\
-0.1773 & -0.0481 & 0.1254 & 0.1836 \\
0.0730 & -0.1047 & -0.1861 & -0.0964
\end{array}\right] \text {, } \\
& B_{c}(0)=\left[\begin{array}{c}
0.0059 \\
0.0133 \\
0.0085 \\
-0.0041
\end{array}\right] \text {, } \\
& C_{c}(0)=\left[\begin{array}{llll}
-0.1794 & -0.1404 & 0.0277 & 0.1704
\end{array}\right] \text {, } \\
& A_{c}(1)=\left[\begin{array}{cccc}
0.5117 & -0.0017 & -0.0517 & -0.0568 \\
-0.0068 & 0.5088 & 0.0601 & 0.0145 \\
-0.0419 & -0.0606 & 0.4383 & 0.0358 \\
0.0615 & 0.0314 & -0.0276 & 0.4012
\end{array}\right] \text {, } \\
& B_{c}(1)=\left[\begin{array}{c}
-0.0035 \\
0.0017 \\
0.0053 \\
0.0040
\end{array}\right], \\
& C_{c}(1)=\left[\begin{array}{llll}
-0.0143 & -0.0731 & -0.0648 & 0.0032
\end{array}\right] .
\end{aligned}
$$

After designing a switched output feedback controller, a suitable $\delta(\sigma)$ should be selected according to the method given above. Solving inequalities (31), (32), (33), and (34) in Theorem 6, we can find that the LMIs are still feasible when $\delta(1)=0.07$ and $\gamma=0.197$ which is smaller than the prescribed 0.95 . This means that the quantization density can be switched to 0.07 when network load is heavy. The network congestion can be reduced. The states evolution of system is shown in Figures 2, 3, 4, and 5. 


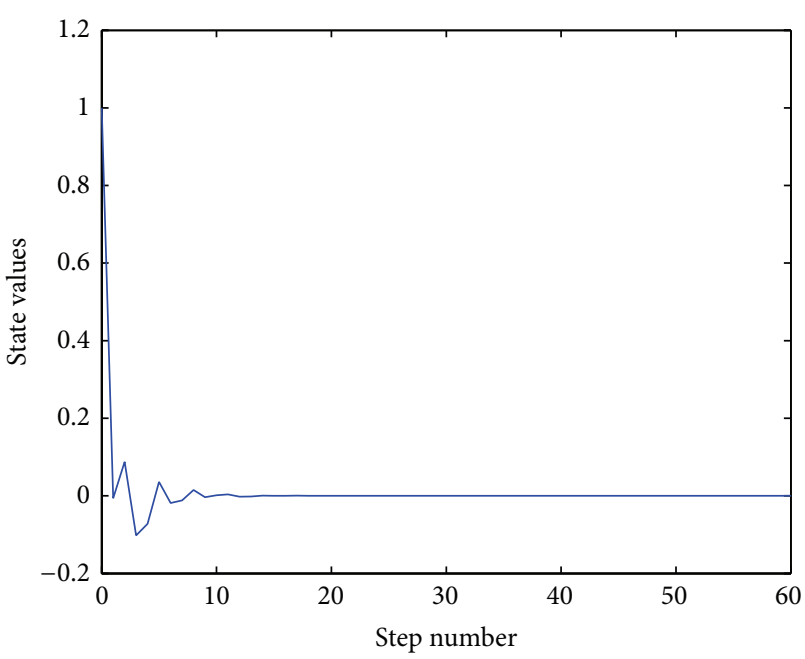

Figure 2: State $X_{1}$.

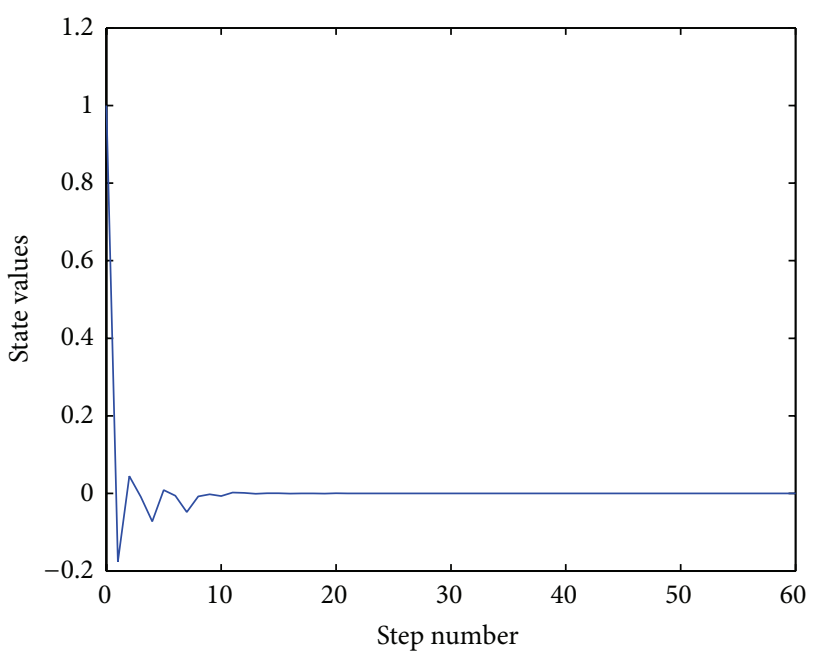

FIgURE 3: State $X_{2}$.

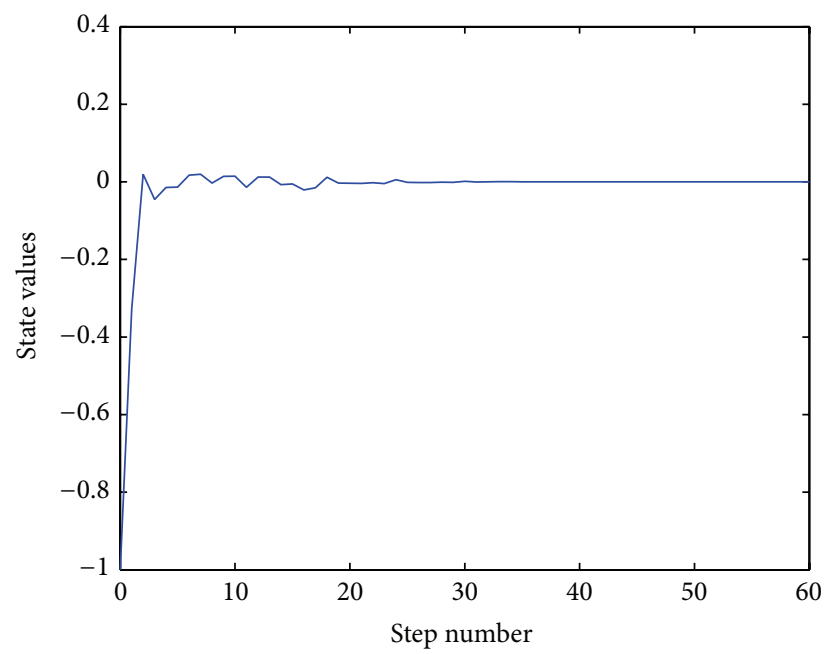

FIgURE 4: State $X_{3}$.

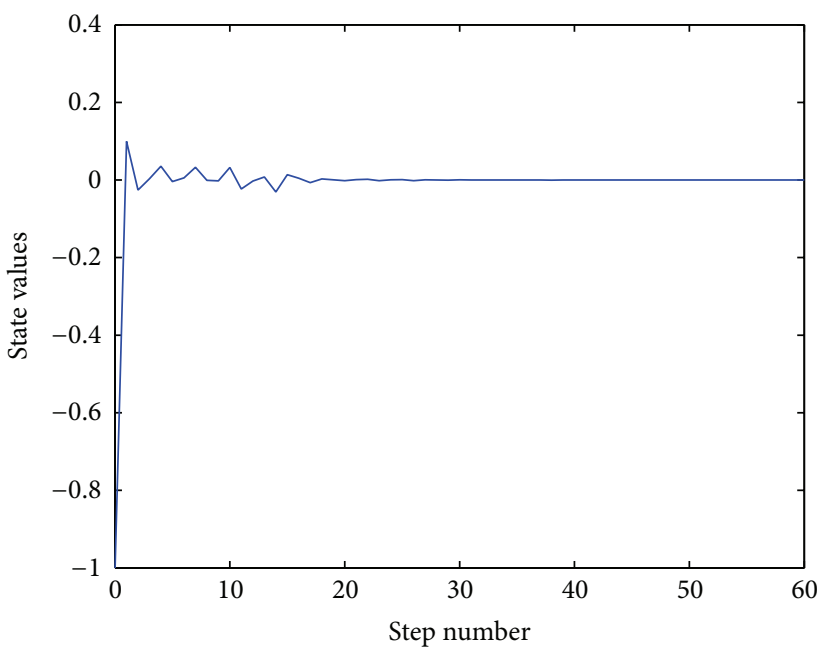

FIgURE 5: State $X_{4}$.

To compare with the case of fixed quantization density, $\delta=0.07$ which corresponds to heavy load is adopted for quantizing data. In this case, network load condition is ignored for design of the controller. A fixed quantization density implies that the load condition cannot be dynamically optimized and the control law can only be computed for the worst load condition. Using Theorem 5, no feasible solution can be found with this density. This indicates that the proposed approach is less conservative.

\section{Conclusions}

The quantitative relationship between quantization density and packet loss number has been studied by switched system approach in this paper. A robust $H_{\infty}$ feedback control law has been derived in terms of bilinear matrix inequalities which can be solved by a cone complement algorithm. A new method for stability analysis of NCS with quantization error and packet drop has been given in the form of Linear Matrix inequalities. An approach to choose suitable quantization density $\delta(d)$ has been presented. The result is less conservative but more flexible since a switched controller and quantization level are used. The effectiveness of the method is illustrated by a simulation example.

\section{Conflict of Interests}

The authors declare that there is no conflict of interests regarding the publication of this paper.

\section{Acknowledgments}

This work is supported by Beijing Municipal Natural Science Foundation (4122075), the National Natural Science Foundation of China under Grants (no. 61004031, no. 61174096, and no. 61104141), and the Fundamental Research Funds for the Central Universities. 


\section{References}

[1] P. Antsaklis and J. Baillieul, "Special issue on technology of networked control systems," Proceedings of the IEEE, vol. 95, no. 1, pp. 5-8, 2007.

[2] Y. Ishido, K. Takaba, and D. E. Quevedo, "Stability analysis of networked control systems subject to packet-dropouts and finite-level quantization," Systems \& Control Letters, vol. 60, no. 5, pp. 325-332, 2011.

[3] W. Yang, M. Liu, and P. Shi, " $H_{\infty}$ filtering for nonlinear stochastic systems with sensor saturation, quantization and random packet losses," Signal Processing, vol. 92, no. 6, pp. 13871396, 2012.

[4] C. Peng and Y.-C. Tian, "Networked $H_{\infty}$ control of linear systems with state quantization," Information Sciences, vol. 177, no. 24, pp. 5763-5774, 2007.

[5] M. Trivellato and N. Benvenuto, "State control in networked control systems under packet drops and limited transmission bandwidth," IEEE Transactions on Communications, vol. 58, no. 2, pp. 611-622, 2010.

[6] H. Song, L. Yu, and W.-A. Zhang, "Networked $H_{\infty}$ filtering for linear discrete-time systems," Information Sciences, vol. 181, no. 3, pp. 686-696, 2011.

[7] K. Tsumura, H. Ishii, and H. Hoshina, "Tradeoffs between quantization and packet loss in networked control of linear systems," Automatica, vol. 45, no. 12, pp. 2963-2970, 2009.

[8] F. Rasool, D. Huang, and S. K. Nguang, "Robust $H_{\infty}$ output feedback control of discrete-time networked systems with limited information," Systems \& Control Letters, vol. 60, no. 10, pp. 845-853, 2011.

[9] D. Liberzon, "A note on stabilization of linear systems using coding and limited communication," in Proceedings of the 41st IEEE Conference on Decision and Control, vol. 1, pp. 836-841, Las Vegas, Nev, USA, 2002.

[10] D. F. Delchamps, "Stabilizing a linear system with quantized state feedback," IEEE Transactions on Automatic Control, vol. 35, no. 8, pp. 916-924, 1990.

[11] R. W. Brockett and D. Liberzon, "Quantized feedback stabilization of linear systems," IEEE Transactions on Automatic Control, vol. 45, no. 7, pp. 1279-1289, 2000.

[12] Y. Zhang and Q. Meng, "Stability of networked control system with quantization," in Proceedings of the Chinese Control and Decision Conference, pp. 559-564, Guilin, China, 2009.

[13] O. C. Imer, S. Yüksel, and T. Başar, "Optimal control of LTI systems over unreliable communication links," Automatica, vol. 42, no. 9, pp. 1429-1439, 2006.

[14] N. Elia and J. N. Eisenbeis, "Limitations of linear remote control over packet drop networks," in Proceedings of the 43rd IEEE International Conference on Decision and Control, vol. 4, pp. 5152-5157, Paradise Island, Bahamas, 2004.

[15] Y. Shi, J. Huang, and B. Yu, "Robust tracking control of networked control systems: application to a networked DC motor," IEEE Transactions on Industrial Electronics, vol. 60, no. 12, pp. 5864-5874, 2013.

[16] H. Li and Y. Shi, "Network-based predictive control for constrained nonlinear systems with two-channel packet dropouts," IEEE Transactions on Industrial Electronics, vol. 61, no. 3, pp. 1574-1582, 2014.

[17] L. Schenato, "Optimal estimation in networked control systems subject to random delay and packet drop," IEEE Transactions on Automatic Control, vol. 53, no. 5, pp. 1311-1317, 2008.
[18] G. N. Nair and R. J. Evans, "Stabilizability of stochastic linear systems with finite feedback data rates," SIAM Journal on Control and Optimization, vol. 43, no. 2, pp. 413-436, 2004.

[19] L. Schenato, B. Sinopoli, M. Franceschetti, K. Poolla, and S. S. Sastry, "Foundations of control and estimation over lossy networks," Proceedings of the IEEE, vol. 95, no. 1, pp. 163-187, 2007.

[20] M. Huang and S. Dey, "Stability of Kalman filtering with Markovian packet losses," Automatica, vol. 43, no. 4, pp. 598607, 2007.

[21] Z. Sun, "Reachability analysis of constrained switched linear systems," Automatica, vol. 43, no. 1, pp. 164-167, 2007.

[22] M. Fu and L. Xie, "The sector bound approach to quantized feedback control," IEEE Transactions on Automatic Control, vol. 50, no. 11, pp. 1698-1711, 2005.

[23] S. Chae, F. Rasool, S. K. Nguang, and A. Swain, "Robust mode delay-dependent $H_{\infty}$ control of discrete-time systems with random communication delays," IET Control Theory \& Applications, vol. 4, no. 6, pp. 936-944, 2009.

[24] H.-L. Zhang, Y.-W. Jing, and S.-Y. Zhang, "Parameter uncertainty-based controller design for networked control systems with active varying sampling period," in Proceedings of the Chinese Control and Decision Conference, pp. 80-85, Shandong, China, July 2008.

[25] D. Xie and Y. Wu, "Stabilisation of time-delay switched systems with constrained switching signals and its applications in networked control systems," IET Control Theory \& Applications, vol. 4, no. 10, pp. 2120-2128, 2010.

[26] L. El Ghaoui, F. Oustry, and M. AitRami, "A cone complementarity linearization algorithm for static output-feedback and related problems," IEEE Transactions on Automatic Control, vol. 42, no. 8, pp. 1171-1176, 1997.

[27] S. Boyd, L. El Ghaoui, E. Feron, and V. Balakrishnan, Linear Matrix Inequalities in System and Control Theory, vol. 15, SIAM, Philadelphia, Pa, USA, 1994. 


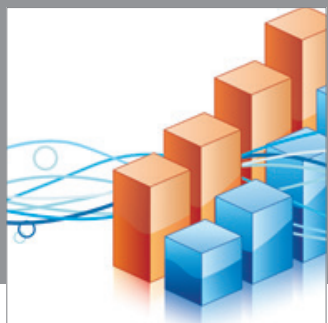

Advances in

Operations Research

mansans

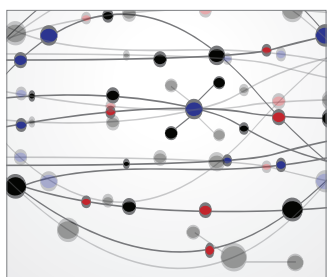

The Scientific World Journal
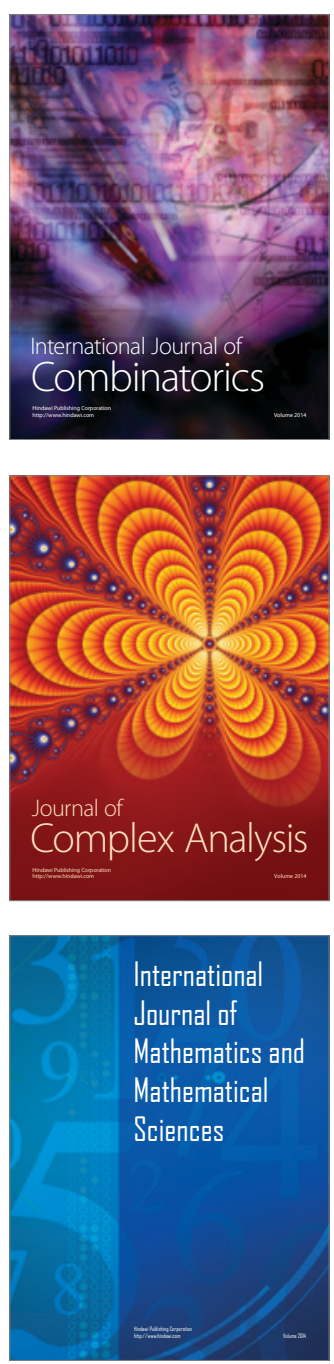
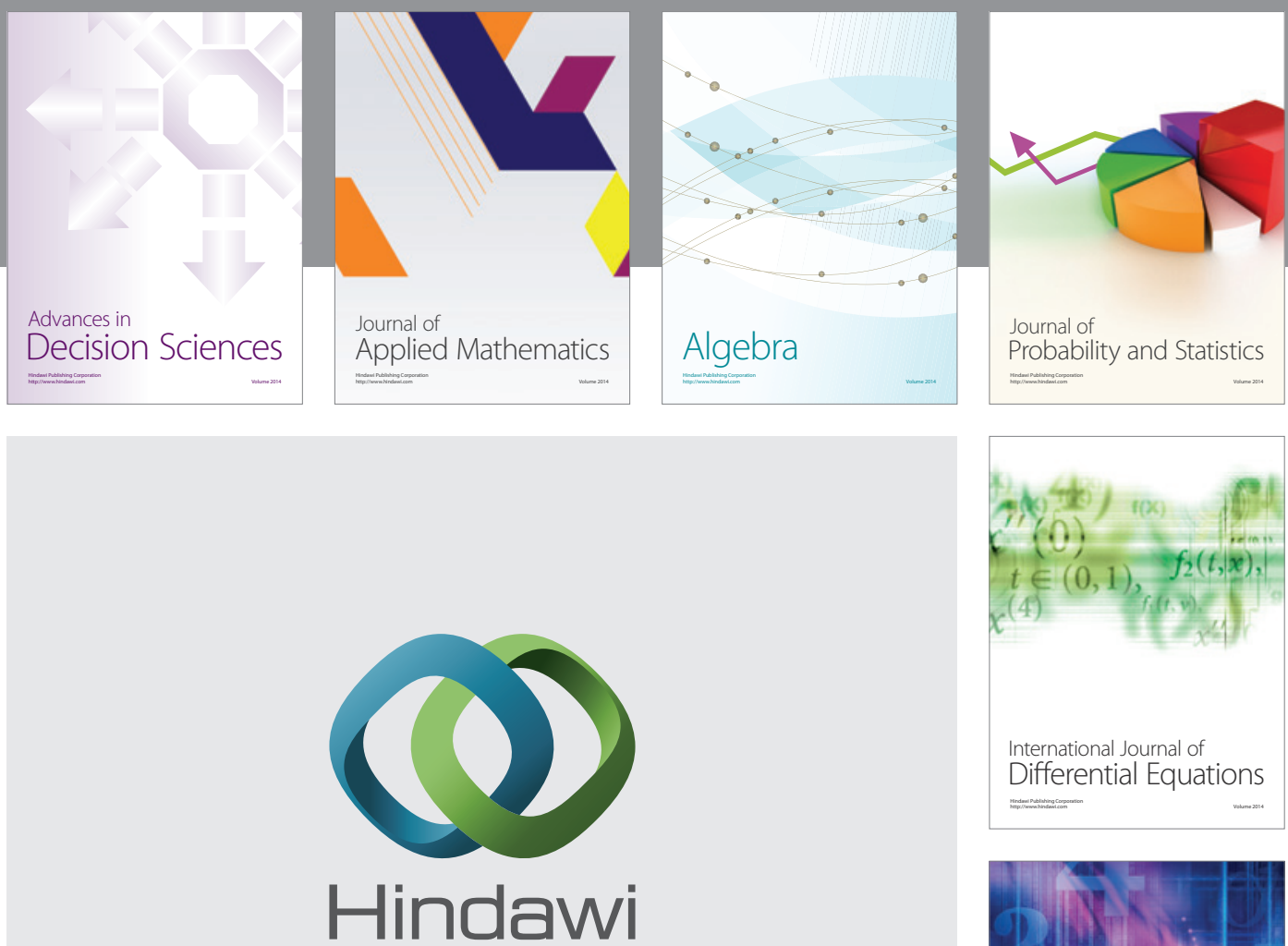

Submit your manuscripts at http://www.hindawi.com
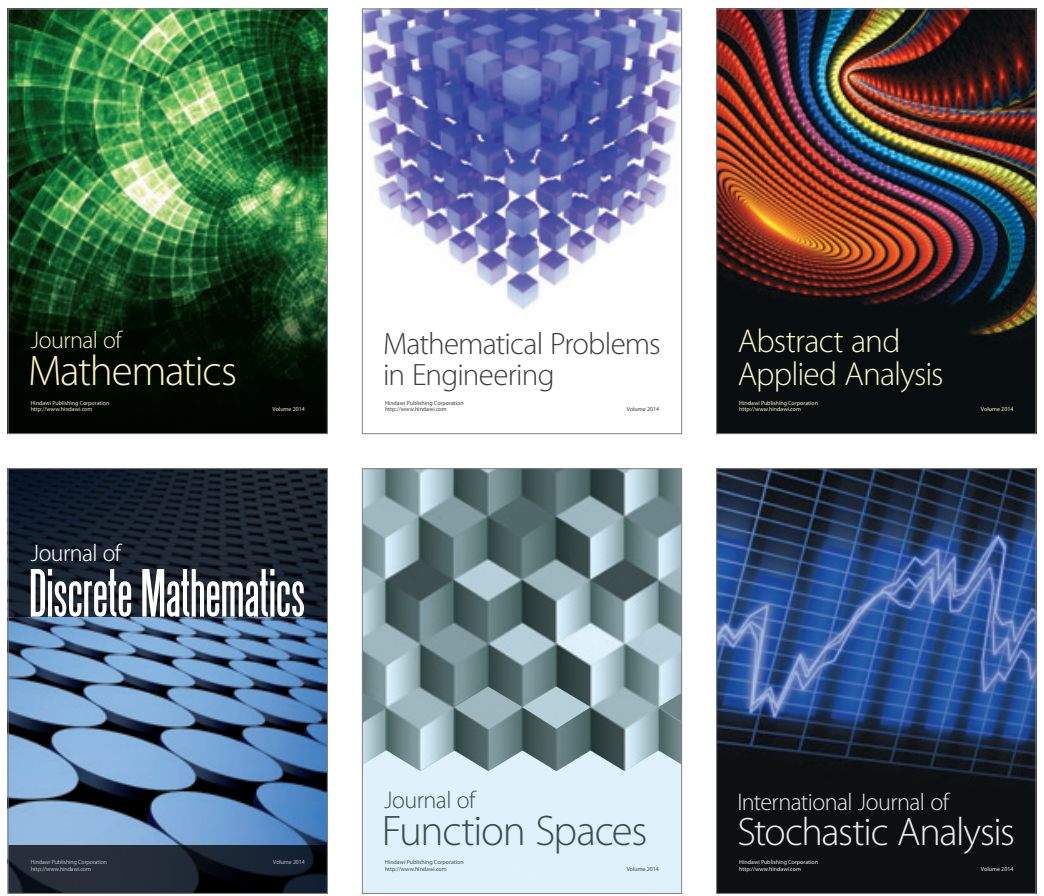

Journal of

Function Spaces

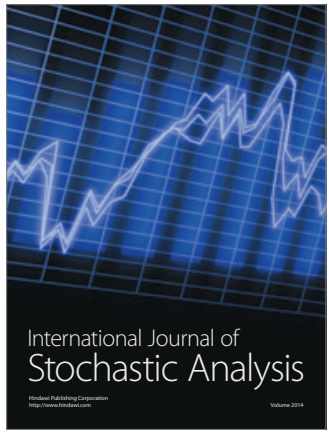

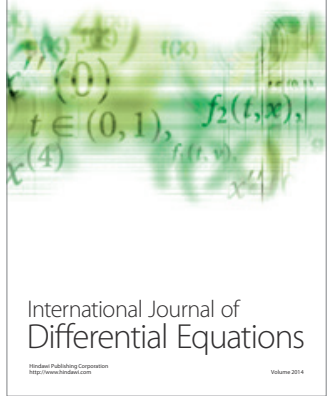
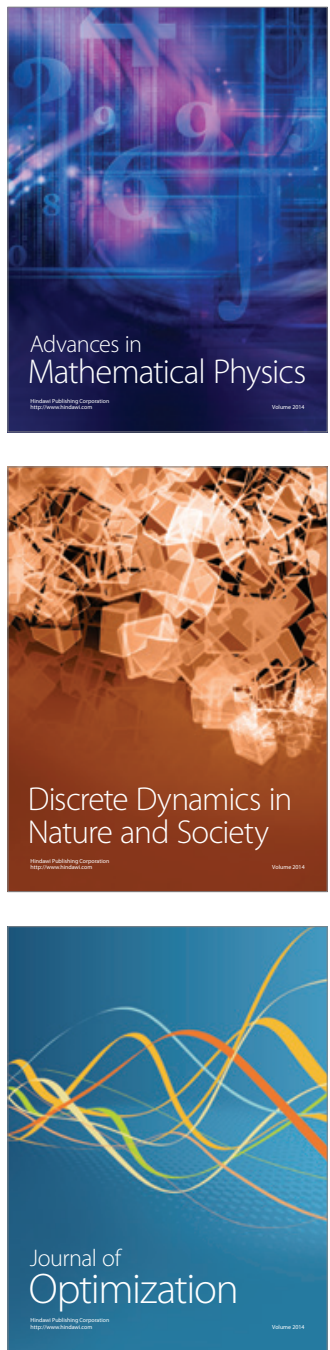